TI 2017-094/II

Tinbergen Institute Discussion Paper

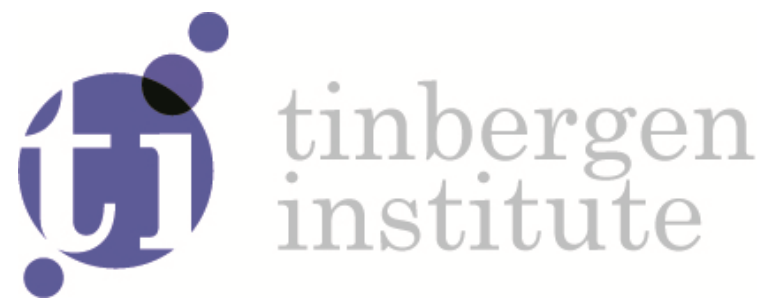

\title{
Interval Solutions for TU-games
}

\author{
Rene (J.R.) van den Brink ${ }^{1}$ \\ Osman Palanci ${ }^{2}$ \\ S. Zeynep Alparslan Gok ${ }^{3}$
}

1: VU Amsterdam; Tinbergen Institute, The Netherlands

2: Suleyman Demirel University, Isparta, Turkey

3: Suleyman Demirel University, Isparta, Turkey 
Tinbergen Institute is the graduate school and research institute in economics of Erasmus University Rotterdam, the University of Amsterdam and VU University Amsterdam.

Contact: discussionpapers@tinbergen.nl

More TI discussion papers can be downloaded at the Tinbergen Site

Tinbergen Institute has two locations:

Tinbergen Institute Amsterdam

Gustav Mahlerplein 117

1082 MS Amsterdam

The Netherlands

Tel.: +31(0)20 5984580

Tinbergen Institute Rotterdam

Burg. Oudlaan 50

3062 PA Rotterdam

The Netherlands

Tel.: +31(0)10408 8900 


\title{
Interval Solutions for TU-games
}

\author{
René van den Brink* \\ Osman Palanci ${ }^{\dagger}$ \\ S. Zeynep Alparslan Gök ${ }^{\ddagger}$
}

\begin{abstract}
Standard solutions for TU-games assign to every TU-game a payoff vector. However, if there is uncertainty about the payoff allocation then we cannot just assign a specific payoff to every player. Therefore, in this paper we introduce interval solutions for TU-games which assign to every TU-game a vector of payoff intervals. Since the solution we propose uses marginal vectors of the interval game, we need to apply a difference operator on intervals. Applying the subtraction operator of Moore (1979), we define an interval solution for TU-games, and we provide an axiomatization.
\end{abstract}

Keywords: Cooperative TU-game, interval game, Moore subtraction, Moore-Shapley interval solution.

\section{Introduction}

Standard solutions for TU-games assign to every TU-game a payoff vector. However, if there is uncertainty about the payoff allocation then we cannot just assign a specific payoff to every player. Therefore, in this paper we introduce interval solutions for TU-games which assign

*Department of Econometrics and Tinbergen Institute, VU University Amsterdam, De Boelelaan 1105, 1081 HV Amsterdam, the Netherlands. E-mail: j.r.vanden.brink@vu.nl

$\dagger$ Department of Business Administration, Süleyman Demirel University, 32260 Isparta, Turkey. E-mail: osmanpalanci@sdu.edu.tr. This author gratefully acknowledges the support of "The Scientific and Technological Research Council of Turkey (TUBITAK)"

${ }^{\ddagger}$ Department of Mathematics, Süleyman Demirel University, 32260 Isparta, Turkey. E-mail: sirmagok@sdu.edu.tr. 
to every TU-game a vector of payoff intervals. To define this type of solution, we first assign to every TU-game an interval game (assigning to every coalition of players an interval worth) and then apply a solution for interval games that extends the Shapley value for TU-games. Specifically, to every coalition we assign the interval worth that has its original worth as lower bound, and its worth in the dual game as upper bound. In game theoretic tradition, the worth of a coalition is defined by a pessimistic approach in the sense that its worth is what the coalition can guarantee irrespective of the behavior of the players outside the coalition. On the other hand, the worth in the dual game equals the worth of the grand coalition minus the worth of the complement coalition in the 'pessimistic' game, and thus can be seen as an optimistic view on the worth of the coalition. In our approach, we put in uncertainty in the game by considering the game and its dual to define the interval worths, so the lower and upper bounds of the interval worths are determined by a pessimistic, respectively optimistic, view on what coalitions can earn. Applying a solution for interval games to this game with uncertainty yields an interval solution for TU-games.

Since the solution we propose uses marginal vectors of the interval game, we need to apply a difference operator on intervals. In the literature several of such difference operators exist, for example that of Moore (1979) and Alparslan Gök, Branzei and Tijs (2009a). It turns out that we cannot apply the subtraction of Alparslan Gök, Branzei and Tijs (2009a). Applying Moore's subtraction we can define an interval solution for TU-games, and we provide an axiomatization. Moore's subtraction is also used by Han, Sun and Xu (2012). However, we argue that their indifference relation is not suitable when exploring uncertainty in TU-games.

The paper is organized as follows. Section 2 contains preliminaries on TU-games and interval games. In Section 3 we introduce and axiomatize two interval solutions for TU-games, which are illustrated in Section 4 by applying them to assignment games. In Section 5 we generalize these interval solutions to a class of interval solutions that are characterized by similar axioms, but differ in the efficiency axiom that is applied. In Section 6, we argue that Han, Sun and Xu (2012) in some sense do the reverse of what we do, in the sense that they assign interval payoffs that are equivalent to single point solutions to interval games. Finally, Section 7 contains concluding remarks. 


\section{Preliminaries}

\subsection{TU-games}

An $n$-person game in characteristic function form (or shortly TU-game) is a pair $(N, v)$ where $N=\{1,2, \ldots, n\}$ is the set of players and $v: 2^{N} \rightarrow$ $\mathbb{R}$ is a characteristic function, such that $v(\emptyset)=0$. Here, $2^{N}$ denotes the set of all subsets of $N$ called coalitions. For each coalition $S$, the real number $v(S)$ is called the worth of $S$ and represents the reward that coalition $S$ can obtain if all its members act together. Since we take the player set fixed, we represent a TU-game $(N, v)$ by its characteristic function $v$. By $\mathcal{G}^{N}$ we denote the $2^{n}-1$ dimensional vector space of all $n$ person games.

A point solution for TU-games is a function which assigns to every game $v \in \mathcal{G}^{N}$, an $n$-dimensional vector which components are the payoffs of the players. One of the most famous solutions for TU-games is the Shapley value (Shapley (1953)) given by

$$
S h(v)=\frac{1}{n !} \sum_{\pi \in \Pi(N)} m^{\pi}(v),
$$

where $\Pi(N)$ is the set of permutations $\pi: N \rightarrow N$ of $N=\{1,2, \ldots, n\}$, and for every $\pi \in \Pi(N)$, the corresponding marginal vector is given by $m_{i}^{\pi}(v)=v\left(P^{\pi}(i) \cup\{i\}\right)-v\left(P^{\pi}(i)\right)$ for each $i \in N$, where $P^{\pi}(i):=$ $\left\{r \in N \mid \pi^{-1}(r)<\pi^{-1}(i)\right\}$, and $\pi^{-1}(i)$ denotes the entrance number of player $i$.

A TU-game $(N, v)$ is superadditive if $v(S \cup T) \geq v(S)+v(T$ for all $S, T \subseteq N$ with $S \cap T=\emptyset$.

We denote the size of a coalition $S \subset N$ by $|S|$. We recall that $\mathcal{G}^{N}$ is a $\left(2^{|N|}-1\right)$ dimensional linear space for which unanimity games form a basis. The unanimity game of $T \in 2^{N} \backslash\{\emptyset\}, u_{T}: 2^{N} \rightarrow \mathbb{R}$, is defined by

$$
u_{T}(S)=\left\{\begin{array}{l}
1, \text { if } T \subseteq S \\
0, \text { otherwise }
\end{array}\right.
$$

It is well-known that a TU-game can be written as a linear combination of unanimity games in a unique way as $v=\sum_{\emptyset \neq T \subseteq N} \Delta_{v}(T) u_{T}$, where the Harsanyi dividends (see Harsanyi (1959)) are given by $\Delta_{v}(T)=$ $\sum_{S \subseteq T}(-1)^{|T|-|S|} v(S)$ for all $T \subseteq N$. The reader is referred to Branzei, Dimitrov and Tijs (2008) and Peters (2008) for a survey on classical $T U$-games.

\subsection{Interval games and interval calculus}

Next, we recall some preliminaries from interval calculus and the theory of cooperative interval games as discussed in Alparslan Gök, Branzei 
and Tijs (2009a, 2009b, 2010).

A cooperative interval game is an ordered pair $(N, w)$ where $N=$ $\{1,2, \ldots, n\}$ is the set of players, and $w: 2^{N} \rightarrow I(\mathbb{R})$, where $I(\mathbb{R})$ is the set of all nonempty, compact intervals in $\mathbb{R}$, is the characteristic function such that $w(\emptyset)=[0,0]$. For each $S \in 2^{N}$, the worth set (or worth interval) $w(S)$ of coalition $S$ in the interval game $(N, w)$ is of the form $w(S)=[\underline{w}(S), \bar{w}(S)]$, where $\underline{w}(S)$ is the minimal worth which coalition $S$ could receive on its own, and $\bar{w}(S)$ is the maximal worth which coalition $S$ could get. The family of all interval games with player set $N$ is denoted by $I G^{N}$.

Let $I, J \in I(\mathbb{R})$ with $I=[\underline{I}, \bar{I}], J=[\underline{J}, \bar{J}]$. Then, $|I|=\bar{I}-\underline{I}$ is called the length of the interval $I$. Also,

(i) $I+J=[\underline{I}+\underline{J}, \bar{I}+\bar{J}]$;

(ii) $\alpha I=[\alpha \underline{I}, \alpha \bar{I}]$, for $\alpha \in \mathbb{R}_{+}$.

By (i) and (ii) we see that $I(\mathbb{R})$ has a cone structure.

In this paper we also need a subtraction operator. In the literature, several subtraction operators can be found. The subtraction operator of Moore (1979) is defined as follows. For intervals $I=[\underline{I}, \bar{I}]$ and $J=[\underline{J}, \bar{J}]$ this difference is defined as $I \ominus J=[\underline{I}-\bar{J}, \bar{I}-\underline{J}]$. For example, let $I=[6,8]$, and $J=[2,5]$, then we have $I \ominus J=[6-5,8-2]=[1,6]$ while $J \ominus I=[2-8,5-6]=[-6,-1]$. Note that this difference is defined for any two intervals. If the intervals are interval payoffs, the difference $I \ominus J$ can be interpreted as follows. Suppose interval $I=[6,8]$ represents some profits that you can earn, but you need to subtract some cost which can be in the interval $J=[2,5]$. Then the best that can happen for you is that you get the highest profit 8 (being the upper bound of the interval $I$ ), and you have to pay the smallest cost 2 (being the lower bound of the interval $J$ ), so your best net earning is $8-2=6$. The worst that can happen for you is that you get the smallest profit 6 (being the lower bound of the interval $I$ ), and you have to pay the highest cost 5 (being the upper bound of the interval $J$ ), so you earn at least $6-5=1$.

An alternative subtraction operator that is used for interval games is that of Alparslan Gök, Branzei and Tijs (2009a). This is defined for intervals $I=[\underline{I}, \bar{I}]$ and $J=[\underline{J}, \bar{J}]$ only if $|I| \geq|J|$, and for such intervals is defined by $I-J=[\underline{I}-\underline{J}, \bar{I}-\bar{J}]$. For the example above, $J-I=[2-6,5-8]=[-4,-3]$, but $I-J$ is not defined. Therefore, this subtraction is defined only for so-called size-monotonic interval games, being interval games $(N, w)$ such that $(N,|w|)$ is a monotonic TU-game, i.e., $|w(S)| \leq|w(T)|$ for all $S \subseteq T \subseteq N$. 
For interval games $w_{1}, w_{2} \in I G^{N}$ we say that $w_{1} \succcurlyeq w_{2}$ if $w_{1}(S) \succcurlyeq$ $w_{2}(S)$, for each $S \in 2^{N}$. For $w_{1}, w_{2} \in I G^{N}$ and $\lambda \in \mathbb{R}_{+}$we define $\left(N, w_{1}+w_{2}\right)$ and $(N, \lambda w)$ by $\left(w_{1}+w_{2}\right)(S)=w_{1}(S)+w_{2}(S)$ and $(\lambda w)(S)=$ $\lambda \cdot w(S)$ for each $S \in 2^{N}$. So, we conclude that $I G^{N}$ endowed with $\succcurlyeq$ is a partially ordered set and has a cone structure with respect to addition and multiplication with non-negative scalars described above. For $w_{1}, w_{2} \in I G^{N},\left(N, w_{1} \ominus w_{2}\right)$ is defined by $\left(w_{1} \ominus w_{2}\right)(S)=w_{1}(S) \ominus$ $w_{2}(S)$ for all $S \subseteq N$, with $\ominus$ the subtraction operator of Moore (1979).

The model of interval cooperative games is an extension of the model of classical TU-games. These games model situations where there is uncertainty about the worths that coalitions can earn, but where at least a lower and upper bound for the worth are known, i.e. the game situation can be described by interval data. However, there is no probability distribution (known) over the possible worths. In that case, interval solutions are useful to solve reward/cost sharing problems where the uncertainty in the game also leads to uncertainty about the payoffs for the players. The components of interval payoff vectors belong to $I(\mathbb{R})$ and represent the interval payoffs of the players. We denote by $I(\mathbb{R})^{N}$ the set of all such interval payoff vectors.

For permutation $\pi \in \Pi(N)$, the interval marginal operators are defined similar as for TU-games ${ }^{1}$ by $m^{\pi}: I G^{N} \rightarrow I(\mathbb{R})^{N}$ as $m_{i}^{\pi}(w)=$ $w\left(P^{\pi}(i) \cup\{i\}\right) \ominus w\left(P^{\pi}(i)\right)$ for each $i \in N$, where we apply the subtraction operator of Moore (1979).

The interval Moore-Shapley value $\Phi^{*}: I G^{N} \rightarrow I(\mathbb{R})^{N}$ is, for each $w \in I G^{N}$, defined by

$$
\begin{aligned}
\Phi^{*}(w) & =\frac{1}{n !} \sum_{\pi \in \Pi(N)} m^{\pi}(w), \\
& =\frac{1}{n !} \sum_{\pi \in \Pi(N)}\left(w\left(P^{\pi}(i) \cup\{i\}\right) \ominus w\left(P^{\pi}(i)\right)\right) .
\end{aligned}
$$

This is one of the values considered by Han, Sun and Xu (2012).

\section{An interval solution for TU-games}

Until so far, the literature studied interval solutions for interval games, and point solutions for classical TU-games. In this paper, we consider interval solutions for TU-games being mappings $f: \mathcal{G}^{N} \rightarrow I(\mathbb{R})^{N}$ assigning to every TU-game an interval payoff vector. These interval payoffs reflect uncertainty in the payoffs to the individual players although

\footnotetext{
${ }^{1}$ Alparslan Gök, Branzei and Tijs (2009a) use another subtraction that is only defined for size-monotonic interval games. In a similar way as below they define an interval Shapley value but using their alternative subtraction operator.
} 
there is no uncertainty about the worths of coalitions. One possibility to define an interval solution is to apply a TU-game solution to the game $v$ and its dual game $v^{d}$, where $v^{d}$ is the dual game of $v$ given by $v^{d}(S)=v(N)-v(N \backslash S)$ for all $S \subseteq N$. So, if the lower bounds are the worths of coalitions in a pessimistic approach, then the upper bounds are determined by taking an optimistic approach with respect to the worths of coalitions. However, if one applies a self-dual ${ }^{2}$ solution $g$, then the intervals are simply containing one element being the payoff assigned by $g$ to $v$. Since we are interested in extending the Shapley value to interval solutions and the Shapley value is self-dual, we cannot follow this approach.

Therefore, for TU-game $v \in \mathcal{G}^{N}$, we define an associated interval game $\left(N, w_{v}\right)$ by assigning to every coalition the interval which is bounded by the worths of the coalition in the game and the dual game, i.e.

$$
w_{v}(S)=\left[v(S), v^{d}(S)\right] \text { for all } S \subseteq N,
$$

Note that, in general the interval game $w$ is not size monotonic since $|w(N)|=\left|\left[v(N), v^{d}(N)\right]\right|=|[v(N), v(N)]|=0$. So, the interval game $w$ is size monotonic only if $|v(S)|=0$ for all $S \subseteq N$, which only is the case when $v$ is an additive game, i.e. when $v(S)=\sum_{i \in S} v(\{i\})$ for all $S \subseteq N$. Therefore, we cannot apply the solution of Alparslan Gök, Branzei and Tijs (2009a). Similar as Han, Sun and Xu (2012), we want to define marginal vectors, but we need to define them to any game, not only size-monotonic games. Therefore, we cannot apply the subtraction operator of Alparslan Gök, Branzei and Tijs (2009a), but we will use the subtraction of Moore (1979). ${ }^{3}$

We first introduce some axioms that we want an interval solution for TU-games to satisfy. The first four axioms are based on classical axioms for TU-game solutions but modified for interval solutions.

Efficiency of interval solutions for interval games means that $\sum_{i \in T} f_{i}(w)=$ $w(N)=[\underline{w(N)}, \overline{w(N)}]$. Since in $w$ as defined in (3) it holds that $w(N)=\overline{w(N)}=v(N)$, efficiency for interval solutions for TU-games requires the sum of the lower bounds as well as upper bounds of the interval payoffs to be equal to $v(N)$. Since this is not suitable, we will modify indifference efficiency as used by Han, Sun and Xu (2012) for interval games, stating that the sum of interval payoffs has the same midpoint as $w(N)$. Since in the interval game defined above $w_{v}(N)$ contains only one element, reflecting that there is no uncertainty about the

\footnotetext{
${ }^{2} \mathrm{~A}$ solution $g$ for TU-games is self-dual if and only if $g(v)=g\left(v^{d}\right)$ for all $v \in \mathcal{G}^{N}$.

${ }^{3}$ Moore's subtraction is also used to define a Shapley value for interval games by Han, Sun and Xu (2012).
} 
worth of the grand coalition in TU-game $v$, we weaken efficiency to midpoint efficiency stating that the midpoints of all interval payoffs add up to $v(N)$.

Axiom 1 (Midpoint Efficiency). For every $v \in \mathcal{G}^{N}$, we have

$$
\sum_{i \in N}\left(\frac{f_{i}(v)+\overline{f_{i}(v)}}{2}\right)=v(N) .
$$

Symmetry states that symmetric players earn the same interval payoff. So, not only their expected payoff is the same, but also the uncertainty around their payoffs. Two players $i, j \in N$ are symmetric in $v$ if $v(S \cup\{i\})=v(\{S \cup\{j\})$ for all $S \subseteq N \backslash\{i, j\}$.

Axiom 2 (Symmetry). If $i, j \in N$ are symmetric players in $v \in \mathcal{G}^{N}$, then $f_{i}(v)=f_{j}(v)$

Note that this is the same as the 'standard' symmetry axiom for TU-game solutions, but here the payoffs are interval payoffs.

A player $i \in N$ is a null player in $v$ if $v(S \cup\{i\})=v(S)$ for all $S \subseteq N \backslash\{i\}$. The null player property of Han, Sun and Xu (2012) for interval games states that a null player earns interval payoffs $[-t, t]$ for some $t \geq 0$. This is the same as equiring that null players earn interval payoffs with midpoint zero. We apply this to TU-games.

Axiom 3 (Null Player Property). If $i \in N$ is a null player in $v \in \mathcal{G}^{N}$, then $\left(\frac{f_{i}(v)+\overline{f_{i}(v)}}{2}\right)=0$.

Linearity is defined as usual.

Axiom 4 (Linearity). For every $v, w \in \mathcal{G}^{N}$ and $a, b \in \mathbb{R}$, we have $f(a v+b w)=a f(v)+b f(w)$.

Besides these 'traditional' (but now in the context of interval solutions for TU-games) axioms, we introduce new axioms that, in some sense, characterize uncertainty in TU-game payoffs by requiring certain upper and lower bounds in extreme cases. First, since there is no uncertainty about the worth of the grand coalition, it seems reasonable that in a superadditive game no player earns more than $v(N)$ (which is the upper bound for the payoffs of the 'grand coalition' $N$ ), i.e. the upper bound of any interval payoff for any player cannot be more than $v(N)$. To have this as a tight upper bound, we define a particular type of game and player for whom this upper bound is reached. We take this to be a veto player in a superadditive game, i.e., a veto player in a superadditive game earns $v(N)$ as upper bound of its payoff. A player $i \in N$ is a veto player in $v$ if $v(S)=0$ for all $S \subseteq N \backslash\{i\}$. 
Axiom 5 (Veto Upper Bound). If $v$ is superadditive and $i \in N$ is a veto player in $v \in \mathcal{G}^{N}$, then $\overline{f_{i}(v)}=v(N)$.

In the previous axiom we considered veto players. Next, we consider games where a particular player becomes a veto player. If player $j \in N$ becomes a veto player in game $v$, this means that all worths of coalitions without player $j$ become zero and we have the game $v^{j}$ given by

$$
v^{j}(S)= \begin{cases}v(S) & \text { if } j \in S, \\ 0 & \text { if } j \notin S .\end{cases}
$$

Suppose that a null player in a superadditive game becomes a veto player. Then the worth of the grand coalition does not change. Therefore, the player who is going to veto is in a stronger position compared to other players, and therefore its upper bound may improve. However, if a null player becomes a veto player then it is not creating more worth but is blocking others. Since blocking does not affect the worst case scenario for a player, we require that its lower bound does not change.

Axiom 6 (Null Becomes Veto Property). If $j \in N$ is a null player in $v \in \mathcal{G}^{N}$ and $v$ is superadditive, then $\underline{f_{j}(v)}=\underline{f_{j}\left(v^{j}\right)}$.

It turns out that the above six axioms characterize a particular interval solution. We first show uniqueness.

Theorem 7. There is a unique interval solution $f: \mathcal{G}^{N} \rightarrow I(\mathbb{R})^{N}$ that satisfies midpoint efficiency, linearity, symmetry, the null player property, veto upper bound and null becomes veto property.

Proof. Consider the unanimity game $u_{T}$ for $T \subseteq N, T \neq \emptyset$. Symmetry implies that there exist numbers $\underline{a}, \bar{a}, \underline{b}, \bar{b} \in \mathbb{R}$ such that $f_{i}\left(u_{T}\right)=[\underline{a}, \bar{a}]$ if $i \in T$, and $f_{i}\left(u_{T}\right)=[\underline{b}, \bar{b}]$ if $i \in N \backslash T$.

The null player property implies that $\underline{b}=-\bar{b}$, i.e. $f_{i}\left(u_{T}\right)=[-\bar{b}, \bar{b}]$ for $i \in N \backslash T$.

By midpoint efficiency and the null player property, we have that

$\sum_{i \in T}\left(\frac{f_{i}\left(u_{T}\right)+\overline{f_{i}\left(u_{T}\right)}}{2}\right)=\frac{t}{2}(\underline{a}+\bar{a})=1$, where $t$ is the number of players in $T$. Thus, with symmetry we have that there is an $a \in \mathbb{R}$ such that $f_{i}\left(u_{T}\right)=\left[\frac{1}{t}-a, \frac{1}{t}+a\right]$ for $i \in T$.

Since $i \in T$ is a veto player in the superadditive game $u_{T}$, by the veto upper bound property we have that $\frac{1}{t}+a=u_{T}(N)=1$, and thus $a=1-\frac{1}{t}=\frac{t-1}{t}$.

So, we determined that $f_{i}\left(u_{T}\right)=\left[\frac{1}{t}-a, \frac{1}{t}+a\right]=\left[\frac{1}{t}-\frac{t-1}{t}, \frac{1}{t}+\frac{t-1}{t}\right]=$ $\left[\frac{2-t}{t}, 1\right]$ for $i \in T$. 
Since $\left(u_{T}\right)^{i}=u_{T} \cup\{i\}$ for $i \in N \backslash T$, the null becomes veto property implies that $\underline{b}=\underline{f}_{i}\left(u_{T \cup\{i\}}\right)=\frac{2-t-1}{t+1}=\frac{1-t}{t+1}$, and thus $f_{i}\left(u_{T}\right)=\left[\frac{1-t}{t+1}, \frac{t-1}{t+1}\right]$ for $i \in N \backslash T$.

So, we have determined $f\left(u_{T}\right)$ for all $\emptyset \neq T \subseteq N$. By linearity we then determined $f(v)$ for all $v \in \mathcal{G}^{N}$.

Next, we explore how the unique solution satisfying the axioms of Theorem 7 looks like. From the proof of the theorem we can see that for unanimity games the unique solution that satisfies the axioms is the solution $\widetilde{f}$ given by

$$
\widetilde{f}_{i}\left(u_{T}\right)= \begin{cases}{\left[\frac{2-t}{t}, 1\right]} & \text { if } i \in T \\ {\left[\frac{1-t}{1+t}, \frac{t-1}{t+1}\right]} & \text { if } i \notin T .\end{cases}
$$

From this we see, using linearity, that for an arbitrary game $v \in \mathcal{G}^{N}$, $\widetilde{f}(v)$ is given by

$$
\widetilde{f}_{i}(v)=\sum_{i \in T} \Delta_{v}(T)\left[\frac{2-t}{t}, 1\right]+\sum_{i \in N \backslash T} \Delta_{v}(T)\left[\frac{1-t}{t+1}, \frac{t-1}{t+1}\right]
$$

In order to prove that this is the solution that is obtained by applying $\Phi^{*}$ (see (1)) to the associated interval game $w_{v}$, we prove the following two lemma's. First, we consider players $i \in T$.

Lemma 8. Let $T \subseteq N, i \in T$, and $S \subseteq N \backslash\{i\}$. Further, let $w=w_{u_{T}}$ be the interval game obtained from $u_{T}$ by (3), i.e. $w(S)=\left[u_{T}(S), u_{T}^{d}(S)\right]$ for all $S \subseteq N$. Then, for $i \in T$, we have $\overline{m_{i}^{S}(w)}=1$ and

$\underline{m_{i}^{S}(w)}= \begin{cases}1, & \text { if } T \backslash\{i\} \subseteq S \text { and } T \cap S=\emptyset \\ 0, & \text { if }[T \backslash\{i\} \subseteq S \text { and } T \cap S \neq \emptyset] \text { or }[T \backslash\{i\} \nsubseteq S \text { and } T \cap S=\emptyset] \\ -1, & \text { if } T \backslash\{i\} \nsubseteq \subseteq \text { and } T \cap S \neq \emptyset\end{cases}$

Proof. Let $T \subseteq N, i \in T$, and $S \subseteq N \backslash\{i\}$, and let $v=u_{T}$. Notice that $u_{T}^{d}(S)=1$ if $S \cap T \neq \emptyset$, and $u_{T}^{d}(S)=0$ otherwise. We distinguish four cases.

Case 1. Suppose that $T \backslash\{i\} \subseteq S$ and $T \cap S=\emptyset$.

Then $v(S \cup\{i\})=v^{d}(S \cup\{i\})=1$ and $v(S)=v^{d}(S)=0$, and thus $m_{i}^{S}(w)=w(S \cup\{i\}) \ominus w(S)=[1,1] \ominus[0,0]=[1,1]$.

Case 2. Suppose that $T \backslash\{i\} \subseteq S$ and $T \cap S \neq \emptyset$.

Then $v(S \cup\{i\})=v^{d}(S \cup\{i\})=v^{d}(S)=1$ and $v(S)=0$, and thus $m_{i}^{S}(w)=w(S \cup\{i\}) \ominus w(S)=[1,1] \ominus[0,1]=[0,1]$. 
Case 3. Suppose that $T \backslash\{i\} \nsubseteq S$ and $T \cap S=\emptyset$.

Then $v^{d}(S \cup\{i\})=1$ and $v(S \cup\{i\})=v(S)=v^{d}(S)=0$, and thus $m_{i}^{S}(w)=w(S \cup\{i\}) \ominus w(S)=[0,1] \ominus[0,0]=[0,1]$.

Case 4. Suppose that $T \backslash\{i\} \nsubseteq S$ and $T \cap S \neq \emptyset$.

Then $v^{d}(S \cup\{i\})=v^{d}(S)=1$ and $v(S \cup\{i\})=v(S)=0$, and thus $m_{i}^{S}(w)=w(S \cup\{i\}) \ominus w(S)=[0,1] \ominus[0,1]=[-1,1]$.

The lemma follows from these four cases.

Next, we consider players $i \in N \backslash T$.

Lemma 9. Let $T \subseteq N, i \in N \backslash T$, and $S \subseteq N \backslash\{i\}$. Further, let $w=w_{u_{T}}$ be the interval game obtained from $u_{T}$ by (3). Then, we have

$$
m_{i}^{S}(w)= \begin{cases}{[0,0]} & \text { if } T \subseteq S \text { or } T \cap S=\emptyset \\ {[-1,1]} & \text { if } T \nsubseteq S \text { and } T \cap S \neq \emptyset\end{cases}
$$

Proof. Let $T \subseteq N, i \in N \backslash T$, and $S \subseteq N \backslash\{i\}$, and let $v=u_{T}$.

We distinguish four cases.

Case 1. Suppose that $T \subseteq S$ and $T \cap S=\emptyset$.

Then $v(S)=v(S \cup\{i\})=1$ and $v^{d}(S)=v^{d}(S \cup\{i\})=0$, and thus $m_{i}^{S}(w)=w(S \cup\{i\}) \ominus w(S)=[1,0] \ominus[0,1]=[0,0]$.

Case 2. Suppose that $T \subseteq S$ and $T \cap S \neq \emptyset$.

Then $v(S)=v(S \cup\{i\})=v^{d}(S)=v^{d}(S \cup\{i\})=1$, and thus $m_{i}^{S}(w)=w(S \cup\{i\}) \ominus w(S)=[1,1] \ominus[1,1]=[0,0]$.

Case 3. Suppose that $T \nsubseteq S$ and $T \cap S=\emptyset$.

Then $v(S)=v(S \cup\{i\})=v^{d}(S)=v^{d}(S \cup\{i\})=0$, and thus $m_{i}^{S}(w)=w(S \cup\{i\}) \ominus w(S)=[0,0] \ominus[0,0]=[0,0]$.

Case 4. Suppose that $T \nsubseteq S$ and $T \cap S \neq \emptyset$. Then, $v(S \cup\{i\})=$ $v(S)=0$ and $v^{d}(S \cup\{i\})=v^{d}(S)=1$, and thus $m_{i}^{S}(w)=w(S \cup\{i\}) \ominus$ $w(S)=[0,1] \ominus[0,1]=[-1,1]$.

The lemma follows from these four cases.

From these lemmas we can prove that the unique solution that satisfies the axioms of Theorem 7 is the solution $\widetilde{f}: \mathcal{G}^{N} \rightarrow I(\mathbb{R})^{N}$ that is obtained by applying the Moore-Shapley interval solution $\Phi^{*}$ to the interval game $w_{v}$ :

$$
\widetilde{f}(v)=\Phi^{*}\left(w_{v}\right) \text { for all } v \in \mathcal{G}^{N},
$$

where $w_{v}$ is the interval game given by (3).

Theorem 10. An interval solution $f: \mathcal{G}^{N} \rightarrow I(\mathbb{R})^{N}$ is equal to $\tilde{f}$ if and only if it satisfies midpoint efficiency, linearity, symmetry, the null player property, veto upper bound and null becomes veto property. 
Proof. By Proposition 7 there is a unique solution satisfying the axioms. Let $v=u_{T}, \emptyset \neq T \subseteq N$, be the unanimity game on $T$, and let $i \in N \backslash T$. Using Lemma 9, we see that $\left.w_{v}\left(P^{\pi}(i) \cup\{i\}\right) \ominus w_{v}\left(P^{\pi}(i)\right)\right)=[-1,1]$ if and only if there is at least one player $j \in T$ with $\pi(j)<\pi(i)$, and at least one player $h \in T$ with $\pi(h)>\pi(i)$. Since the number of permutations of $T \cup\{i\}$ where $\min _{j \in T} \pi(j)<\pi(i)<\max _{h \in T} \pi(h)$ is equal to $(t-1) t$ !, by (1) we have $\Phi_{i}^{*}\left(w_{v}\right)=\frac{1}{n !} \sum_{\pi \in \Pi(N)}\left(w_{v}\left(P^{\pi}(i) \cup\{i\}\right) \ominus w_{v}\left(P^{\pi}(i)\right)\right)=$ $\frac{(t-1) t !}{(t+1) !}[-1,1]=\frac{t-1}{t+1}[-1,1]=\left[\frac{1-t}{t+1}, \frac{t-1}{t+1}\right]=\widetilde{f}_{i}\left(u_{T}\right)$ by $(4)$.

Next, consider $i \in T$. By Lemma 8 we know that $\overline{m_{i}^{S}\left(w_{v}\right)}=1$. By symmetry, there is an $a \in \mathbb{R}$ such that $m_{i}^{S}(w)=a$ for all $i \in T$. By midpoint efficiency, it then holds that $\overline{\sum_{i \in T} \frac{a+1}{2}}=\frac{(a+1) t}{2}=1$, and thus $a=\frac{2}{t}-1=\frac{2-t}{t}=\widetilde{f}_{i}\left(u_{T}\right)$ by $(4)$.

$\widetilde{f}$.

With linearity of $\Phi^{*}$ and (5), it follows that the axioms characterize

We show logical independence by the following alternative solutions.

1. Consider the interval solution $f$ given by $f(v)=\sum_{T \subseteq N, T \neq \emptyset} \Delta_{v}(T) f\left(u_{T}\right)$, where for unanimity games $u_{T}, \emptyset \neq T \subseteq N$, the interval payoffs are given by

$$
f_{i}\left(u_{T}\right)=\left\{\begin{array}{l}
{[0,1] \text { if } i \in T} \\
{[0,0] \text { if } i \in N \backslash T .}
\end{array}\right.
$$

This interval solution satisfies linearity, symmetry, the null player property, the veto upper bound property and the null becomes veto property. It does not satisfy midpoint efficiency.

2. The interval solution $f$ given by $f(v)=\widetilde{f}\left(\sum_{T \subseteq N} \Delta_{v}(T) u_{E(v)}\right)$ where $E(v)$ is the set of nonnull players in $v$, satisfies midpoint efficiency, symmetry, the null player property, veto upper bound and null becomes veto property. It does not satisfy linearity.

3. Consider a given positive weight vector $\lambda \in \mathbb{R}_{++}^{N}$, and the solution $f$ given by $f(v)=\sum_{T \subset N, T \neq \emptyset} \Delta_{v}(T) f\left(u_{T}\right)$, where for unanimity games $u_{T}, \emptyset \neq T \subseteq N$, the interval payoffs are given by

$$
f_{i}\left(u_{T}\right)=\left\{\begin{array}{cl}
{\left[\frac{\lambda_{i}}{\sum_{j \in T} \lambda_{j}}(2-t), 1\right]} & \text { if } i \in T \\
{\left[\frac{\lambda_{i}}{\sum_{j \in T} \lambda_{j}}(1-t), \sum_{j \in T} \lambda_{j}(t-1)\right]} & \text { if } i \in N \backslash T
\end{array}\right.
$$


This interval solution satisfies midpoint efficiency, linearity, the null player property, the veto upper bound property and the null becomes veto property. It does not satisfy symmetry.

4. The interval solution $f$ given by $f_{i}(v)=[v(N), v(N)]$ for all $i \in N$, satisfies midpoint efficiency, linearity, symmetry, veto upper bound and null becomes veto property. It does not satisfy the null player property.

5. Consider the interval solution $f$ given by $f(v)=\sum_{T \subseteq N, T \neq \emptyset} \Delta_{v}(T) f\left(u_{T}\right)$, where for unanimity games $u_{T}, \emptyset \neq T \subseteq N$, the interval payoffs are given by

$$
f_{i}\left(u_{T}\right)=\left\{\begin{array}{l}
{\left[0, \frac{2}{t}\right] \text { if } i \in T} \\
{[0,0] \text { if } i \in N \backslash T .}
\end{array}\right.
$$

This interval solution satisfies midpoint efficiency, linearity, symmetry, the null player property, and the null becomes veto property. It does not satisfy the veto upper bound property.

6. The interval solution $\widehat{f}$ that we discuss next satisfies midpoint efficiency, linearity, symmetry, the null player property, and veto upper bound property. It does not satisfy the null becomes veto property.

Expression (5) makes clear that the interval payoff of player $i$ consists of a part that it earns from unanimity games it belongs to, and part of those where it is a null player.

Since null players earn an interval payoff with midpoint zero, letting a player share only in unanimity games it belongs to, we obtain the alternative midpoint efficient solution $\widehat{f}$ given by

$$
\widehat{f}_{i}(v)=\sum_{i \in T} \Delta_{v}(T)\left[\frac{2-t}{t}, 1\right]
$$

It turns out that this solution satisfies all axioms of Theorem 7 except the null becomes veto property. It is characterized by replacing the null becomes veto property by a stronger null player property which requires zero payoffs for null players (and nut just zero midpoints).

Axiom 11 (Strong Null Player Property). If $i \in N$ is a null player in $v \in \mathcal{G}^{N}$ then $f_{i}(v)=[0,0]$. 
Theorem 12. An interval solution $f: \mathcal{G}^{N} \rightarrow I(\mathbb{R})^{N}$ is equal to the solution $\widehat{f}$ if and only if it satisfies midpoint efficiency, linearity, symmetry, the strong null player property and the veto upper bound property.

Proof. It is obvious that $\widehat{f}$ satisfies linearity, symmetry, the strong null player proerty and the veto upper bound property.

Midpoint efficiency follows by linearity and the fact that since, for $\emptyset \neq T \subseteq N$ and $v=u_{T}$, we have $\sum_{i \in N}\left(\frac{\widehat{f_{i}(v)+}+\widehat{\hat{f}_{i}(v)}}{2}\right)=\sum_{i \in T} \frac{\frac{2-t}{t}+1}{2}=$ $t\left(\frac{2-t+t}{2 t}\right)=1$, where the first equality follows from the strong null player property.

To prove uniqueness, again consider the unanimity game $u_{T}$ for $T \subseteq$ $N, T \neq \emptyset$. The strong null property implies that $f_{i}\left(u_{T}\right)=[0,0]$ for all $i \in N \backslash T$. Similar as in the proof of Theorem 7, it follows from midpoint efficiency, symmetry and the veto upper bound property that $f_{i}\left(u_{T}\right)=\left[\frac{2-t}{t}, 1\right]$ for $i \in T$.

So, we have determined $f\left(u_{T}\right)$ for all $\emptyset \neq T \subseteq N$. By linearity we then determined $f(v)$ for all $v \in \mathcal{G}^{N}$.

We show logical independence by the following alternative solutions. These are modifications of the solutions showing logical independence of the axioms in Theorem 10, but taking account of other null player payoffs.

1. Consider the interval solution $f$ given by $f(v)=\sum_{T \subseteq N, T \neq \emptyset} \Delta_{v}(T) f\left(u_{T}\right)$, where for unanimity games $u_{T}, \emptyset \neq T \subseteq N$, the interval payoffs are given by

$$
f_{i}\left(u_{T}\right)=\left\{\begin{array}{l}
{[0,1] \text { if } i \in T} \\
{[0,0] \text { if } i \in N \backslash T .}
\end{array}\right.
$$

This interval solution satisfies linearity, symmetry, the strong null player property and the veto upper bound property. It does not satisfy midpoint efficiency.

2. The interval solution $f$ given by $f(v)=\tilde{f}\left(\sum_{T \subset N} \Delta_{v}(T) u_{E(v)}\right)$ where $E(v)$ is the set of nonnull players in $v$, satisfies midpoint efficiency, symmetry, the strong null player property and the veto upper bound property. It does not satisfy linearity.

3. Consider a given positive weight vector $\lambda \in \mathbb{R}_{++}^{N}$, and the solution $f$ given by $f(v)=\sum_{T \subseteq N, T \neq \emptyset} \Delta_{v}(T) f\left(u_{T}\right)$, where for unanimity 
games $u_{T}, \emptyset \neq T \subseteq N$, the interval payoffs are given by

$$
f_{i}\left(u_{T}\right)=\left\{\begin{array}{cl}
{\left[\frac{\lambda_{i}}{\sum_{j \in T} \lambda_{j}}(2-t), 1\right]} & \text { if } i \in T, \\
{[0,0]} & \text { if } i \in N \backslash T .
\end{array}\right.
$$

This interval solution satisfies midpoint efficiency, linearity, the strong null player property, and the veto upper bound property. It does not satisfy symmetry.

4. The interval solution $\tilde{f}$ satisfies midpoint efficiency, linearity, symmetry, and the veto upper bound property. It does not satisfy the strong null player property.

5. Consider the interval solution $f$ given by $f(v)=\sum_{T \subseteq N, T \neq \emptyset} \Delta_{v}(T) f\left(u_{T}\right)$, where for unanimity games $u_{T}, \emptyset \neq T \subseteq N$, the interval payoffs are given by

$$
f_{i}\left(u_{T}\right)=\left\{\begin{array}{l}
{\left[0, \frac{2}{t}\right] \text { if } i \in T} \\
{[0,0] \text { if } i \in N \backslash T .}
\end{array}\right.
$$

This interval solution satisfies midpoint efficiency, linearity, symmetry, and the strong null player property. It does not satisfy the veto upper bound property.

From the expressions of the solutions $\widetilde{f}$ and $\widehat{f}$ above, we see that both satisfy the desirable property that in every game, every player earns an interval which midpoint coincides with its Shapley value in the TU-game. So, both solutions extend the Shapley value in the sense that the interpretation of the Shapley value is still that of an expected payoff, but they differ in the way they bring in uncertainty in the payoffs of the players, i.e. different lengths of the interval payoffs.

This is desirable since we are interested in extending the Shapley value in the sense that we respect the Shapley value as expected payoffs, but want to acknowledge uncertainty in payoffs. Therefore, we could state the following axiom which requires that all interval payoffs have the Shapley value value of the corresponding player as midpoint.

Axiom 13 (Shapley-midpoint property). For every $v \in \mathcal{G}^{N}$, we have $\frac{f_{i}(v)+\overline{f_{i}(v)}}{2}=S h_{i}(v)$ for all $i \in N$.

By (4) and (6), we have the following corollary.

Corollary 14. The solutions $\tilde{f}$ and $\widehat{f}$ satisfy the Shapley midpoint property. 
Since the Shapley midpoint property implies midpoint efficiency as well as the null player property, in Theorem 7 we can replace these axioms. $^{4}$

Corollary 15. An interval solution $f: \mathcal{G}^{N} \rightarrow I(\mathbb{R})^{N}$ is equal to $\tilde{f}$ if and only if it satisfies the Shapley midpoint property, linearity, symmetry, the veto upper bound and the null becomes veto property.

Reviewing the axioms, we can say that linearity and symmetry are standard axioms, also for solutions without uncertainty. The other axioms can be related to either refering to the expected payoffs or the uncertainty around these payoffs. Midpoint efficiency and the (strong) null player property are refering to the expected payoffs, while the veto upper bound property and the null becomes veto property refer to the uncertainty of the payoffs.

\section{An application: Assignment games}

In this section we illustrate the two extensions of the Shapley value that are introduced and axiomatized in the previous section by an assignment game (see Shapley and Shubik (1972)). A motivation for the Shapley value as expected payoffs for assignment games is given in van den Brink and Pintér (2015). Consider an assignment situation with two buyers (players 1 and 2) and one seller (player 3). Assume that player 3 has a house for sale for which he has reservation value 0 . The two buyers want to buy the house, and their reservation values are 1, respectively 2 . Then, the coalition values of the assignment game are

$$
\begin{gathered}
v(\{1\})=v(\{2\})=v(\{3\})=v(\{1,2\})=0 \\
v(\{1,3\})=1 \text { and } v(\{2,3\})=v(\{1,2,3\})=2 .
\end{gathered}
$$

\footnotetext{
${ }^{4}$ Something similar can be done for $\widehat{f}$, but then we need to keep the strong null player property since that is not implied by midpoint efficiency.
} 
The Harsanyi dividends are $\Delta_{v}(\{1,3\})=1, \Delta_{v}(\{2,3\})=2, \Delta_{v}(N)=$ -1 , and $\Delta_{v}(S)=0$ otherwise. From this we find,

$$
\begin{aligned}
\widetilde{f}_{1}(v) & =\sum_{\substack{T \subseteq N \\
1 \in T}} \Delta_{v}(T)\left[\frac{2-t}{t}, 1\right]+\sum_{\substack{T \subseteq N \\
1 \notin T}} \Delta_{v}(T)\left[\frac{1-t}{t+1}, \frac{t-1}{t+1}\right] \\
& =\left[\frac{2-2}{2}, 1\right]+2\left[\frac{1-2}{2+1}, \frac{2-1}{2+1}\right]+(-1)\left[\frac{2-3}{3}, 1\right] \\
& =[0,1]+2\left[-\frac{1}{3}, \frac{1}{3}\right]+(-1)\left[-\frac{1}{3}, 1\right] \\
& =[0,1]+\left[-\frac{2}{3}, \frac{2}{3}\right]+\left[-1, \frac{1}{3}\right] \\
& =\left[-\frac{5}{3}, 2\right] .
\end{aligned}
$$

Similarly, we find

$$
\tilde{f}_{2}(v)=\left[-\frac{4}{3}, \frac{8}{3}\right] \text { and } \widetilde{f}_{3}(v)=\left[-1, \frac{10}{3}\right] .
$$

As we see, the three midpoints equal the Shapley value of the assignment game $S h(v)=\left(\frac{1}{6}, \frac{2}{3}, \frac{7}{6}\right)$. Additionally, $\widetilde{f}$ assigns the players uncertainty around their payoffs, respectively leading to an interval payoff of length $\left|\widetilde{f}_{1}(v)\right|=\frac{11}{3},\left|\widetilde{f}_{2}(v)\right|=\frac{12}{3}$ and $\left|\widetilde{f}_{3}(v)\right|=\frac{13}{3}$.

For $\widehat{f}(v)$ we find

$$
\begin{aligned}
\widehat{f_{1}}(v) & =\sum_{\substack{T \subseteq N \\
1 \in T}} \Delta_{v}(T)\left[\frac{2-t}{t}, 1\right] \\
& =\left[\frac{2-2}{2}, 1\right]+(-1)\left[\frac{2-3}{3}, 1\right] \\
& =\left[-1, \frac{4}{3}\right]
\end{aligned}
$$

and similarly,

$$
\widehat{f_{2}}(v)=\left[-1, \frac{7}{3}\right] \text { and } \widehat{f}_{3}(v)=\left[-1, \frac{10}{3}\right] .
$$

Again, the midpoints of the interval payoffs equal the Shapley value of $v$, but for the two buyers there is less uncertainty. 


\section{A class of interval solutions for TU-games}

At the end of Section 3 we saw that the two interval solutions $\widetilde{f}$ and $\widehat{f}$ extend the Shapley value in the sense that to every TU-game they assign interval payoffs to the players such that for each player the midpoint of its interval payoff equals the Shapley value of the game, see Corollary 15. We can extend any linear TU-game solution in this way, i.e. any linear TU-game solution can be extended to an interval solution that is characterized by the axioms of Corollary 14 but with an appropriately modified midpoint property. Recall that $f$ denotes a generic interval solution, and $g$ denotes a generic point solution for TU-games.

Axiom 16 (g-midpoint property). Let $g$ be a linear solution for $T U$ games. For every $v \in \mathcal{G}^{N}$, we have $\frac{f_{i}(v)+\overline{f_{i}(v)}}{2}=g_{i}(v)$ for all $i \in N$.

This can be done only for linear TU-game solutions. For linear TUgame solution $g$, let the interval solution $f^{g}$ be given by

$f_{i}^{g}(v)=\sum_{i \in T} \Delta_{v}(T)\left[2 g_{i}\left(u_{T}\right)-1,1\right]+\sum_{i \in N \backslash T} \Delta_{v}(T)\left[2 g_{i}\left(u_{T \cup\{i\}}\right)-1,1-2 g_{i}\left(u_{T \cup\{i\}}\right)\right]$.

Replacing the Shapley midpoint property in Corollary 15 by the $g$ midpoint property characterizes a corresponding interval solution.

Theorem 17. Let $g$ be a linear TU-game solution satisfying the null player property. An interval solution $f: \mathcal{G}^{N} \rightarrow I(\mathbb{R})^{N}$ is equal to $f^{g}$ if and only if it satisfies the $g$-midpoint property, linearity, symmetry, veto upper bound and null becomes veto property.

Proof. The proof is similar to the proof of Theorem 7 .

It is straightforward to verify that $f^{g}$ satisfies the axioms. To prove uniqueness, let $f$ be an interval solution satisfying the properties. By linearity, $f$ can be written as

$$
f_{i}(v)=\sum_{i \in T} \Delta_{v}(T) f_{i}\left(u_{T}\right)+\sum_{i \in N \backslash T} \Delta_{v}(T) f_{i}\left(u_{T}\right) .
$$

Consider the unanimity game $u_{T}$ for $T \subseteq N, T \neq \emptyset$. By symmetry and the veto upper bound property, there exist numbers $a, b \in \mathbb{R}$ such that $f_{i}\left(u_{T}\right)=[a, 1]$ if $i \in T$. By symmetry and the $g$-midpoint property $f_{i}\left(u_{T}\right)=[-b, b]$ if $i \in N \backslash T$.

By the $g$-midpoint property we then have that $\frac{a+1}{2}=g_{i}\left(u_{T}\right)$, which is equivalent to $a=2 g_{i}\left(u_{T}\right)-1$. The veto becomes null player proerty 
then implies that $-b=2 g_{i}\left(u_{T \cup\{i\}}\right)-1$, which is equivalent to $b=$ $1-2 g_{i}\left(u_{T \cup\{i\}}\right)$.

This implies that $f=f^{g}$ given by (7).

Note that instead of requiring $g$ to satisfy the null player property for TU-game solutions, we could require $f$ to satisfy the null player property for interval solutions.

As an example, we can take $g=B a$ as the Banzhaf value, see Banzhaf (1965), Dubey and Shapley (1979) and Owen (1975). For unanimity games, the Banzhaf value is given by

$$
B a_{i}\left(u_{T}\right)=\left\{\begin{array}{ll}
\frac{1}{2^{t-1}}, & \text { if } i \in T \\
0 & \text {, if } i \notin T
\end{array} .\right.
$$

Example 18. Taking $w_{v}$ as the interval game associated with $v$, the interval Banzhaf value, characterized by the axioms in Theorem 17, is defined as

$$
\begin{aligned}
f_{i}^{B a}(v) & =\sum_{\substack{T \subseteq N \\
i \in T}} \Delta_{v}(T)\left[2 B a_{i}\left(u_{T}\right)-1,1\right]+\sum_{\substack{T \subseteq N \\
i \notin T}} \Delta_{v}(T)\left[2 B a_{i}\left(u_{T \cup\{i\}}\right)-1,1-2 B a_{i}\left(u_{T \cup\{i\}}\right)\right] \\
& =\sum_{\substack{T \subseteq N \\
i \in T}} \Delta_{v}(T)\left[2 \frac{1}{2^{t-1}}-1,1\right]+\sum_{\substack{T \subseteq N \\
i \notin T}} \Delta_{v}(T)\left[2 \frac{1}{2^{t}}-1,1-2 \frac{1}{2^{t}}\right] \\
& =\sum_{\substack{T \subseteq N \\
i \in T}} \Delta_{v}(T)\left[\frac{1-2^{t-2}}{2^{t-2}}, 1\right]+\sum_{\substack{T \subseteq N \\
i \notin \bar{T}}} \Delta_{v}(T)\left[\frac{1-2^{t-1}}{2^{t-1}}, \frac{2^{t-1}-1}{2^{t-1}}\right]
\end{aligned}
$$

\section{A comment on interval games: midpoint equiva- lence}

Although this paper is on TU-games, in this section we briefly comment on interval games. Han, Sun and Xu (2012) characterterize a class of solutions for interval games by the axioms of indifference efficiency, the indifference null player property, symmetry and additivity for interval games. In this paper, we applied interval games to define interval solutions for TU-games. In our case there is uncertainty about the payoff allocation, but there is no uncertainty about the worth of coalitions.

Han, Sun and Xu (2012) define an indifference relation on intervals by saying that two intervals are equivalent if and only if they have the same midpoint, i.e. for $I, J \subset I(\mathbb{R})$

$$
I \sim J \Leftrightarrow \frac{I+\bar{I}}{2}=\frac{J+\bar{J}}{2}
$$


and for $w, w^{\prime} \in I \mathcal{G}^{N}$, we have

$$
w \sim w^{\prime} \Leftrightarrow w(S) \sim w^{\prime}(S) \text { for all } S \subseteq N .
$$

If two games with the same midpoint are equivalent, when defining a solution for interval games, one can apply the following axiom.

Axiom 19 (Midpoint equivalence). For $w, w^{\prime} \in I G^{N}$, if $w \sim w^{\prime}$ then $f(w)=f\left(w^{\prime}\right)$.

With this axiom, we can use interval versions of 'classical' TU-game axioms to characterize the Shapley value.

Axiom 20 (Efficieny). For every $w \in I G^{N}$, we have $\sum_{i \in N} f_{i}(w)=$ $w(N)$.

Axiom 21 (Symmetry). For every $w \in I G^{N}$, if $i, j \in N$ are symmetric players in $w$ then $f_{i}(w)=f_{j}(w)$.

Axiom 22 (Null player property). For every $w \in I G^{N}$, if $i \in N$ is a null player in $w$ then $f_{i}(w)=[0,0]$.

Axiom 23 (Linearity). For every $w, w^{\prime} \in I G^{N}$, we have $f\left(w+w^{\prime}\right)=$ $f(w)+f\left(w^{\prime}\right)$.

It turns out that the axioms above characterize the interval solution that assigns to every interval TU-game just the Shapley value of its midpoint game, i.e. for $w \in I G^{N}$, we define the solution $\varphi^{S h}$ given by

$$
\varphi^{S h}(w)=\left[S h\left(v_{w}\right), \operatorname{Sh}\left(v_{w}\right)\right],
$$

where the midpoint game $v_{w} \in \mathcal{G}^{N}$ is given by

$$
v_{w}(S)=\frac{w(S)+\overline{w(S)}}{2} \text { for all } S \subseteq N .
$$

Theorem 24. An interval solution $g: I \mathcal{G}^{N} \rightarrow I(\mathbb{R})^{N}$ is equal to the Shapley value $\varphi^{S h}$ if and only if it satisfies efficiency, the null player property, symmetry, linearity and midpoint equivalence.

Proof. It is straightforward to verify that $\varphi^{S h}$ satisfies the axioms. Uniqueness follows since every interval game $v \in I \mathcal{G}^{N}$ is equivalent to the game $w_{v}(S)=\left[\frac{v(S)+\overline{v(S)}}{2}, \frac{v(S)+\overline{v(S)}}{2}\right]$ and therefore by midpoint equivalence, $\varphi^{S h}(v)=\varphi^{S h}\left(w_{v}\right)$, where $\varphi^{S h}\left(w_{v}\right)$ is determined by the other axioms similar as the axiomatization of the Shapley value in classical TU-games. 
This result is not satisfying because we wanted to express uncertainty about the payoffs in the solution. A reason is the indifference relation ((6)) that is underlying midpoint equivalence. As mentioned, Han, Sun and $\mathrm{Xu}$ (2012) introduced this indifference relation saying that intervals are ranked according to their midpoint. Applying this to interval games, essentially one says that an interval game is equivalent to the classical game that assigns to every coalition the midpoint of its interval worth, and thus, under this indifference relation, assigning the Shapley value makes sense as an expected value, but the indifference relation ignores the uncertainty in the game. On the other hand, in this paper we introduced uncertainty in the payoffs in games where there is no uncertainty about the worths of coalitions.

Midpoint equivalence and the equivalence relation of Han, Sun and $\mathrm{Xu}$ (2012) in some sense assume players to be risk neutral. Instead, if players are risk-averse then the equivalence relation might be $I \sim J \Longleftrightarrow$ $\underline{I}=\underline{J}$. In this case the players evaluate a game by the worst coalitions they belong to can do. Similar, we can define indifference relations for risk-loving players by, e.g. $I \sim J \Longleftrightarrow \bar{I}=\bar{J}$.

\section{Concluding remarks}

In this paper, interval solutions are obtained for TU games. In the literature, there exist classical solutions for classical TU games and interval solutions for cooperative interval games in literature. Different from the existing literature, we have constructed interval solutions for classical TU-games.

First of all, from classical TU games by choosing the coalition value as the lower bound and the dual coalition value as the upper bound we have constructed a cooperative interval games. After that, we have obtained generalized Shapley values which provide some axioms on cooperative interval games.

In this study, Moore's subtraction operator (Moore (1979)) is used different from the special subtraction operator Alparslan Gök, Branzei and Tijs (2009a) used for cooperative interval games. Further, an application is done on assignment games. Finally, the interval Banzaf value is introduced by using Moore's subtraction operator.

Interval solutions for TU-games reflect uncertainty about the payoff allocations in situations where there is no uncertainty in the worths of coalitions. This uncertainty can have several reasons. One reason can be that there are externalities from payoff allocations. For example, if a player prefers egalitarian outcomes, then large payoff differences among players might lead to a negative externality for this player. On the other hand, a 'competitive' player might derive positive externalities 
from a payoff allocation where it earns more than other players. In specific allocation problems such as the assignment game that is discussed in Section 4, the 'low valuation' buyer 1 might derive a positive effect on its utility payoff when it earns a positive payoff, although the 'high valuation' buyer 2 and the seller 3 can generate the total worth of the grand coalition by themselves. On the other hand, buyer 1 might have a negative externality on its utility payoff if he gets nothing from the surplus, although he has 'leverage' in the game. When the utility assigned to payoff allocations is not known, this might be modeled by interval payoffs. ${ }^{5}$

For future studies, we want to extend our research to other OR games by using Moore's subtraction operator. Similarly, we plan to introduce different linear interval solutions for classical TU games.

\section{References}

[1] Alparslan Gök, S.Z., Branzei, R., Tijs, S., 2009a. Convex Interval Games. Journal of Applied Mathematics and Decision Sciences, Article ID 342089, 14 pages.

[2] Alparslan Gök, S.Z., Miquel, S., Tijs, S., 2009b. Cooperation under interval uncertainty. Mathematical Methods of Operations Research, 69, 99-109.

[3] Alparslan Gök, S.Z., Branzei, R., Tijs, S., 2010. The interval Shapley value: an axiomatization. Central European Journal of Operations Research, 18(2), 131-140.

[4] Banzhaf, J.F., 1965. Weighted Voting Doesnít Work: A Mathematical Analysis. Rutgers University Law Review, 19, 317-343.

[5] Branzei, R., Dimitrov, D., Tijs, S., 2008. Models in Cooperative Game Theory. Springer-Verlag, 204 pages, Berlin.

[6] Brink, R. van den, and Pintér, M., 2015. On Axiomatizations of the Shapley Value for Assignment Games. Journal of Mathematical Economics, 60, 110-114.

[7] Dubey, P., Shapley, L.S., 1979. Mathematical properties of the Banzhaf power index. Mathematics of Operations Research 4(2), 99-131.

[8] Han, W., Sun, H., Xu, G., 2012. A new approach of cooperative interval games: the interval core and Shapley value revisited. Operations Research Letters, 40(6), 462-468.

[9] Harsanyi, J.C., (1959), A Bargaining Model for Cooperative nPerson Games. In Contributions to the Theory of Games IV (A.W.

\footnotetext{
${ }^{5} \mathrm{~A}$ utility foundation of the Shapley value as a von Neumann-Morgenstern expected utility function is given by Roth (1977).
} 
Tucker and R.D. Luce, Eds.), pp. 325- 355. Princeton: Princeton UP.

[10] Moore, R., 1979. Methods and Applications of Interval Analysis. SIAM Studies in Applied Mathematics, 190 pages, Philadelphia.

[11] Owen, G., 1975. On the core of linear production games. Mathematical Programming, 9(1), 358-370.

[12] Peters, H., 2008. Game Theory: A Multi-Leveled Approach. Springer-Verlag, Berlin Heidelberg, 494 pages, Berlin.

[13] Roth, A.E., 1977. The Shapley value as a von NeumannMorgenstern utility. Econometrica, 45 (3), 657-664.

[14] Shapley, L.S., 1953. A value for $n$-person games. Annals of Mathematics Studies, 28, 307-317.

[15] Shapley, L.S., Shubik M., 1972. The assignment game I: the core, International Journal of Game Theory, 1(2), 111-130. 